\title{
Nódulos tireoidianos - uma abordagem diagnóstica
}

\author{
Thyroid nodules - a diagnostic approach \\ Nódulos tiroideos - un enfoque de diagnóstico
}

Luma de Oliveira Morais $^{1 *}$, Giovanna Costa Fagundes Vieira ${ }^{1}$, Ane Caroline Leal Monteiro Botelho ${ }^{1}$, Emanuelle Alves Lacerda ${ }^{1}$, Ézio Júnio Gonçalves Nunes ${ }^{2}$, Lenilson Prates da Silva ${ }^{2}$, Moara Halanna Barbosa $^{1}$, Bárbara Gonçalves Mascarenhas ${ }^{3}$, Franciany Gonçalves Mascarenhas ${ }^{4}$, Kamila Lima Abrantes de Matos $^{5}$, Célia Carvalho Cavalcante ${ }^{6}$, Francine Ribeiro Potros ${ }^{7}$, Marianne Caldeira de Faria Santiago ${ }^{1}$, Ana Clara Cruz Braga ${ }^{5}$.

\section{RESUMO}

Objetivo: Realizar uma revisão de literatura a respeito dos nódulos tireoidianos, considerando a importância da abordagem diagnóstica dos mesmos. Métodos: Foi realizada uma pesquisa de revisão de literatura consultando artigos científicos indexados nas bases eletrônicas LILACS, SCIELO, MEDLINE publicados no período de 2003 a 2018, incluindo estudos disponíveis em sua totalidade, gratuitos, publicados nos últimos quinze anos, nos idiomas Português, Espanhol e Inglês. Revisão Bibliográfica: A avaliação dos nódulos tireoidianos tem como objetivo principal diferenciar lesões benignas e malignas tendo em vista o potencial tratamento conservador dos nódulos benignos e a necessidade de intervenção mais agressiva nas lesões malignas (tireoidectomia). Anamnese e exame físico deverão ser obtidos para caracterizar o nódulo e avaliar presença de adenomegalia cervical. Inicialmente deve ser feita a dosagem do TSH; se aumentado, a cintilografia da tireoide está indicada. A ultrassonografia de tireoide é o exame mais frequentemente utilizado para confirmar achados do exame clínico e guiar a punção por agulha fina (PAAF). Tomografia Computadorizada e Ressonância Magnética são raramente indicadas pois não permitem diferenciar lesões malignas e benignas. A PAAF é o melhor método para realizar esta diferenciação, sendo considerado exame padrão-ouro. Conclusão: É importante estudar nódulos tireoidianos, visto que o conhecimento sobre o assunto favorece o diagnóstico precoce daqueles sugestivos de malignidade, melhorando o prognóstico do paciente.

Palavras-chave: Doenças da Glândula Tireoide, Nódulo da Glândula Tireoide, Consenso.

\footnotetext{
ABSTRACT

Objective: To carry out a review of the literature regarding thyroid nodules, considering the importance of the diagnostic approach of the same. Methods: A review of the literature was carried out by consulting scientific articles indexed in the electronic databases LILACS, SCIELO, MEDLINE published in the period from 2003 to 2018, including free studies published in the last fifteen years in Portuguese, Spanish and English. Literature

${ }^{1}$ Residência Médica em Medicina de Família e Comunidade - Universidade Estadual de Montes Claros, Porteirinha-MG. *E-mail: lumamorais90@hotmail.com

${ }^{2}$ Especialização em Saúde Coletiva com concentração em Atenção Básica - Saúde da Família - Universidade Federal da Bahia.

${ }^{3}$ Especialização em Gestão do Cuidado em Saúde da Família - Universidade Federal de Minas Gerais.

${ }^{4}$ Faculdade Uniatenas - Paracatu-MG.

${ }^{5}$ Instituto de Ciências da Saúde - ICS.

${ }^{6}$ Especialização em Dermatologia - Faculdade IPEMED de Ciências Médicas.

${ }^{7}$ Universidade Estadual de Montes Claros.
}

SUBMETIDO EM: 1/2019

ACEITO EM: 2/2019

PUBLICADO EM: 5/2019

REAS/EJCH | Vol.Sup.23 | e402 | DOI: https://doi.org/10.25248/reas.e402.2019 Página 1 de 7 
Review: The aim of the evaluation of thyroid nodules is to differentiate between benign and malignant lesions in view of the potential conservative treatment of benign nodules and the need for more aggressive intervention in malignant lesions (thyroidectomy). Anamnesis and physical examination should be obtained to characterize the nodule and evaluate the presence of cervical adenomegaly. Initially the TSH should be dosed; if elevated, thyroid scintigraphy is indicated. Thyroid ultrasonography is the most frequently used test to confirm clinical examination findings and guide fine needle puncture (FNAB). Computed tomography and magnetic resonance imaging are rarely indicated because they do not allow differentiation between malignant and benign lesions. FNAB is the best method to perform this differentiation, being considered a gold standard examination. Conclusion: It is important to study thyroid nodules, since the knowledge about the subject favors the early diagnosis of those suggestive of malignancy, improving the prognosis of the patient.

Key words: Thyroid Diseases Thyroid, Nodule Thyroid Neoplasms, Consents.

\section{RESUMEN}

Objetivo: Realizar una revisión de literatura acerca de los nódulos tiroideos, considerando la importancia del abordaje diagnóstico de los mismos. Métodos: Se realizó una revisión bibliográfica de la investigación mediante la consulta de artículos científicos indexados en bases de datos electrónicas LILACS, SciELO, MEDLINE publicados en el período de 2003 a 2018, incluidos los estudios disponibles en su totalidad, libres, publicado en los últimos quince años, en portugués, español y Ingles. Revisión Bibliográfica: La evaluación de los nódulos tiroidianos tiene como objetivo principal diferenciar lesiones benignas y malignas teniendo en vista el potencial tratamiento conservador de los nódulos benignos y la necesidad de intervención más agresiva en las lesiones malignas (tiroidectomía). La anamnesis y el examen físico deben ser obtenidos para caracterizar el nódulo y evaluar la presencia de adenomegalia cervical. Inicialmente se debe realizar la dosificación del TSH; si se aumenta, se indica la centellografía de la tiroides. La ecografía de tiroides es el examen más frecuentemente utilizado para confirmar los hallazgos del examen clínico y guiar la punción por aguja fina (PAAF). La tomografía computarizada y la resonancia magnética son raramente indicadas porque no permiten diferenciar lesiones malignas y benignas. La PAAF es el mejor método para realizar esta diferenciación, siendo considerado examen patrón-oro. Conclusión: Es importante estudiar nódulos tiroideos, ya que el conocimiento sobre el tema favorece el diagnóstico precoz de aquellos sugestivos de malignidad, mejorando el pronóstico del paciente.

Palabras clave: Enfermedades de la Tiroides, Nódulo Tiroideo Neoplasias de la Tiroides, Consenso.

\section{INTRODUÇÃO}

Nódulos tireoidianos são encontrados com facilidade na prática clínica, com uma prevalência aproximada de $10 \%$ na população adulta, havendo prevalência maior quando em estudos que levem em consideração o diagnóstico por ultrassonografia (US). Representam a principal manifestação de uma série de doenças tireoidianas e a maioria dos acometidos tem doença benigna (MAIA AL, et al., 2007; OLIVA EG, 2012; PECCIN S, et al., 2003; ROSÁRIO PW, et al., 2013). Estudos de pessoas oriundas de áreas com suplementação suficiente de iodo na alimentação mostram que cerca de $4 \%$ a $7 \%$ das mulheres e $1 \%$ dos homens adultos apresentam nódulo tireoidiano palpável ao exame físico. Em estudos com ultrassonografia essa prevalência chega a $68 \%$, sendo essas frequências mais elevadas em mulheres idosas (MAIA AL, et al., 2007; OLIVA EG, 2012; ROSÁRIO PW, et al., 2013).

Apesar de a maioria dos nódulos tireoidianos ser benigna, a abordagem diagnóstica visa excluir a malignidade, que ocorre em 5 a 15\% dos casos (sendo que desses 95\% corresponde ao carcinoma bem diferenciado), uma vez que em alguns indivíduos a neoplasia de tireoide pode constituir uma patologia agressiva e desafiadora para os estudos atuais (COOPER DS, et al., 2009; KIMURA ET, et al., 2011; MAIA AL, et al., 2007; ROSÁRIO PW, et al., 2013; WARD LS, 2013). 
A incidência atual do câncer de tireoide é próxima de 24 casos por 100.000 habitantes, mas essa taxa vem aumentando nos últimos anos, ocupando o lugar da quarta neoplasia maligna mais frequente nas mulheres brasileiras (ROSÁRIO PW, et al., 2013). A última estimativa global indicou a ocorrência de aproximadamente 300.000 novos casos desse câncer, correspondendo a $1.150 \mathrm{em}$ homens e $8.050 \mathrm{em}$ mulheres (CRISTO AP, et al., 2016). Esse número crescente se deve principalmente ao aumento dos diagnósticos de pequenos carcinomas papilíferos (ROSÁRIO PW, et al., 2013). Apesar do aumento de incidência, a taxa de mortalidade por essa causa permaneceu praticamente inalterada (MAIA AL, et al., 2007).

Nos últimos anos tem-se visto atualizações a respeito do diagnóstico e seguimento dos nódulos tireoidianos, uma vez que constitui uma importante e comum doença em nosso meio. O diagnóstico precoce de lesões da tireoide permite tratamentos mais específicos e curativos. Duas entidades clássicas [Sociedade Brasileira de Endocrinologia e Metabologia (SBEM) e Associação Americana de Tireoide (ATA)] publicaram recomendações pertinentes ao assunto e fundamentais para a prática clínica.

O presente estudo tem como objetivo realizar uma revisão a respeito do tema, considerando a importância da abordagem diagnóstica dos nódulos tireoidianos visto que é preciso excluir precocemente o câncer de tireoide, doença potencialmente grave da tireoide. O trabalho é de grande valia já que encontramos poucas revisões de literatura envolvendo a temática, constituindo uma valiosa leitura frente a uma clínica sugestiva desta predominante patologia tireoidiana.

\section{METODOLOGIA}

Neste estudo foi realizada uma pesquisa de revisão de literatura, aqui entendida como um estudo exploratório da produção do conhecimento acerca de um assunto ou tema, verificando assim, o conhecimento produzido e obtendo elementos para analisá-lo. Como fonte de dados foram consultados artigos científicos indexados nas bases eletrônicas LILACS, SCIELO, MEDLINE publicados em periódicos, no período de 2003 a 2018.

Tendo como etapas para sua elaboração: definição do objetivo; estabelecimento de critérios de inclusão e exclusão para a seleção da amostra; definição das informações a serem extraídas dos artigos selecionados; análise, e discussão dos resultados.

Os critérios de inclusão definidos foram: estudos disponíveis em sua totalidade, gratuitos, publicados nos últimos quinze anos, nos idiomas Português, Espanhol e Inglês. Foram excluídos da busca inicial capítulos de livros, artigos que não abordassem o tema proposto, resumos, textos incompletos e relatos técnicos.

\section{REVISÃO DE LITERATURA}

A avaliação dos nódulos tireoidianos tem como objetivo principal diferenciar lesões benignas e malignas tendo em vista o potencial tratamento conservador dos nódulos benignos e a necessidade de intervenção mais agressiva nas lesões malignas (tireoidectomia) (OLIVA EG, 2012).

Diante de um nódulo tireoidiano na prática clínica o profissional deve ser obter uma anamnese e um exame físico detalhado, com vistas principalmente à definição das características do nódulo e à avaliação da presença de adenomegalia cervical. Apesar de a história clínica, na maioria das vezes, não ser sensível ou específica, existem alguns dados que se associam a um maior risco de malignidade (Tabela 1) (MAIA AL, et al., 2007; ROSÁRIO PW, et al., 2013).

Tanto o consenso brasileiro da Sociedade Brasileira de Endocrinologia e Metabologia quanto a Associação Americana de Tireoide concordam que na abordagem inicial dos nódulos tireoidianos deve ser feita a dosagem do TSH (Hormônio Estimulante da Tireoide) (COOPER DS, et al., 2009; MAIA AL, et al., 2007; PAZFILHO G, et al., 2013; ROSÁRIO PW, et al., 2013). Se esta avaliação inicial revelar hiperfunção da glândula, ainda que subclínica, a cintilografia da tireoide (com iodo radioativo, de preferência) está indicada para determinar se o nódulo é hipercaptante (absorção do marcador é maior que a tireoide normal circundante), 
isocaptante (absorção do marcador é igual a tireoide circundante) ou hipocaptante (absorção menor que a tireoide normal circundante). Um estudo recente evidenciou que valores elevados de TSH estão relacionados com maior risco de malignidade (COOPER DS, et al., 2009; MAIA AL, et al., 2007; ROSÁRIO PW, et al., 2013). Cerca de $10 \%$ dos pacientes que possuem nódulos solitários apresentam TSH suprimido e nódulo hipercaptante, e nesses casos, a punção por agulha fina (PAAF) não é necessária, uma vez que esses nódulos em sua grande maioria são benignos. (MAIA AL, et al., 2007; ROSÁRIO PW, et al., 2013).

Quadro 1. Dados sugestivos de malignidade do nódulo tireoidiano

\begin{tabular}{|l|l|}
\hline \multicolumn{1}{|c|}{ História clínica sugestiva de malignidade } & \multicolumn{1}{|c|}{ Exame físico sugestivo de malignidade } \\
\hline Sexo masculino; & Nódulo com rápido crescimento ou volumoso com \\
Paciente com idade menor que 20 anos ou superior & sintomas compressivos; \\
a 70 anos; & Nódulo de consistência endurecida, aderido a \\
História de exposição à radiação ou radioterapia & planos profundos, pouco móvel; \\
cervical na infância ou adolescência; & Nódulo associado a paralisia ipsilateral de corda \\
Diagnóstico prévio de câncer de tireoide tratado & vocal; \\
com tireoidectomia parcial; & Presença de linfonodomegalia cervical. \\
História familiar (parente de primeiro grau) de & \\
câncer de tireoide; & \\
Síndromes hereditárias como Neoplasia Endócrina & \\
Múltipla Tipo 2 (NEM 2), síndrome de Werner, & \\
Complexo de Carney, polipose adenomatosa & \\
familiar, entre outras. & \\
\hline
\end{tabular}

Fonte: Próprio autor, 2019; ROSÁRIO PW, et al., 2013.

A dosagem sérica de calcitonina tem uma interpretação controversa sobre seus resultados e sobre seu custo/benefício, sendo, talvez, mais interessante em paciente acima de 40 anos com nódulos pequenos (MAIA AL, et al., 2007). A ATA aborda o uso da dosagem de calcitonina para detectar hiperplasia de células $C$ e carcinoma medular da tiroide em estágios iniciais e consequente melhoria da sobrevida, no entanto, ainda permanecem questões não resolvidas de sensibilidade, especificidade e custo/benefício (COOPER DS, et al., 2009). Já a dosagem de Tireoglobulina ( $\mathrm{Tg}$ ) é um teste relativamente insensível no diagnóstico de neoplasia maligna da tireoide (COOPER DS, et al., 2009; MAIA AL, et al., 2007; PAZ-FILHO G, et al., 2013; ROSÁRIO PW, et al., 2013).

A ultrassonografia de tireoide é o exame mais frequentemente utilizado para confirmar achados do exame clínico, para medir nódulos e para guiar PAAF. A observação de características dos nódulos durante a realização da US pode fornecer informações importantes sobre os mesmos. Alguns aspectos ultrassonográficos como: conteúdo sólido, hipoecogenicidade, margens irregulares, microcalcificações, halo periférico ausente ou descontínuo, padrões de fluxo ao estudo com Doppler (predominante ou exclusivamente central), diâmetro anteroposterior maior que o anterior e detecção de adenomegalia cervical foram associados ao câncer de tireoide. Em um estudo recente foram considerados nódulos suspeitos aqueles que apresentavam microcalcificações ou hipoecogenicidade ou halo ausente (combinação em paralelo) com sensibilidade de $81 \%$ e especificidade de $70 \%$. Os bons resultados observados com as combinações de características ultrassonográficas em paralelo tornam o exame um método elegível para a avaliação dos nódulos tireoidianos. Entretanto, a sua utilidade deve ser analisada comparativamente à PAAF, método 
padrão utilizado para a avaliação diagnóstica de câncer em nódulos de tireoide (KIMURA ET, et al., 2011; MAIA AL, et al., 2007; PECCIN S, et al., 2003; ROSÁRIO PW, et al., 2013).

Outros exames disponíveis são a Tomografia Computadorizada (TC) e Ressonância Magnética (RM), porém são raramente indicadas na avaliação de nódulos tireoidianos pois não diferenciam lesões malignas e benignas. No entanto, são úteis na avaliação de bócios mergulhantes ou da compressão e invasão de estruturas adjacentes, como a traqueia. A Tomografia com emissão de prótons utilizando a fluoro deoxiglicose-18F (Positron Emission Tomography-Fluoro-deoxiglucose) (FDG-PET) é útil na diferenciação de lesões malignas e benignas, no entanto é um método pouco acessível e de custo elevado (COOPER DS, et al., 2009; KIMURA ET, et al., 2011; MAIA AL, et al., 2007; ROSÁRIO PW, et al., 2013).

A Cintilografia com isótopos, como já mencionada, revela se o nódulo é hipercaptante, com ou sem supressão extranodular, e apesar de também não diferenciar nódulo benigno de maligno, chama atenção pela possibilidade de nodulação maligna. Ainda, os exames está indicado na presença de nódulo cuja citologia sugere neoplasia folicular naquele paciente com TSH baixo ou diminuído (KIMURA ET, et al., 2011; ROSÁRIO PW, et al., 2013).

A PAAF é o melhor método para diferenciar nódulos malignos e benignos, tendo sido utilizada como padrão-ouro, demonstrando ser seguro, fácil execução, baixo custo, baixo índice de complicação e com boa acurácia (COOPER DS, et al., 2009; MAIA AL, et al., 2007; OLIVA EG, 2012; ROSÁRIO PW, et al., 2013); exibe altas taxas de sensibilidade (65 a 98\%) e especificidade (72 a 100\%), com baixas taxas de falso positivo (0 a 7\%) e falso negativo (1 a 11\%) reduzindo as tireoidectomias desnecessárias, e os custos em mais de $25 \%$.

Por outro lado, a utilização de uma técnica adequada, médicos experientes para realização da PAAF e para análise do material aspirado são fatores determinantes no aumento da acurácia do método, guiando uma terapêutica apropriada (OLIVA EG, 2012).

Ward e Kloos (2013) ressalta a importância da PAAF, uma vez que, anteriormente ao advento da mesma, os nódulos tireoidianos eram rotineiramente encaminhados para cirurgia diagnóstica devido ao possível risco de malignidade (5 a 15\%). Sendo assim, a PAAF diminuiu a tireoidectomia diagnóstica pela metade, devido ao fato de que a maioria dos resultados do material aspirado é conclusivamente diagnosticado como citologia benigna.

Devem ser puncionados nódulos maiores que $1,0 \mathrm{~cm}$. Para nódulos menores que $1,0 \mathrm{~cm}$ a indicação de PAAF são para os com características ultrassonográficas sugestivas de malignidade ou história clínica de risco, exceto quando há suspeita de nódulo funcionante. A SBEM e a ATA se assemelham sobre quando biopsiar os nódulos. De acordo com a ATA, todos os nódulos sólidos $\geq 1,0-1,5 \mathrm{~cm}$ devem ser biopsiados (> $1,0 \mathrm{~cm}$ se hipoecoicos; $\geq 1,0-1,5 \mathrm{~cm}$ se iso-ou hiperecoico), enquanto a SBEM recomenda a biópsia de todos os nódulos $\geq 1 \mathrm{~cm}$ caso sejam hipoecoicos; se os nódulos sólidos forem iso ou hiperecoicos, a biópsia deve ser realizada se $\geq 1,5 \mathrm{~cm}$, mesmo na ausência de achados ultrassonográficos anormais (COOPER DS, et al., 2009; KIMURA ET, et al., 2011; PAZ-FILHO G, et al., 2013; ROSÁRIO PW, et al., 2013).

Para nódulos de tireoide menores que $1,0 \mathrm{~cm}$ diagnosticados por ultrassonografia, cujos pacientes não possuam fatores de risco para neoplasia maligna (população de baixo risco), é recomentado o controle com exame de imagem de ultrassonografia periódico (anual), acrescido do controle da função hormonal tireoidiana (KIMURA ET, et al., 2011).

Quanto ao uso do US para guiar a PAAF, A SBEM sugere que a PAAF guiada por ultrassom só é útil quando as amostras iniciais são inadequadas, ao passo que a ATA recomenda especificamente o uso de ultrassom quando os nódulos não são palpáveis, são predominantemente císticos, ou estão posteriormente localizados no lobo da tireoide e para os casos quando a PAAF precisa ser repetida devido ao um resultado não diagnóstico. Noutros estudos, ficou evidente que a PAAF guiada por US diminui as taxas de resultados inadequados e falso-negativos e que melhora a acurácia, a sensibilidade e o valor preditivo positivo (COOPER DS, et al., 2009; PAZ-FILHO G, et al., 2013; ROSÁRIO PW, et al., 2013). 
Após uma conferência multidisciplinar promovida pelo National Cancer Institute ( $\mathrm{NCl}$ - USA) foi proposta uma classificação de acordo com o resultado da citolopatologia conhecida como Sistema Bethesda, em que guia os laudos citopatológigos de tireoide ao dividir em categorias, a saber: I: Amostra não diagnóstica, II: Benigno, III: Atipias/Lesão folicular de significado indeterminado, IV: Suspeito para neoplasia folicular ou neoplasia folicular, V: Suspeito para malignidade e VI: Maligno (CRISTO AP, et al., 2016; ROSÁRIO PW, et al., 2013).

O tratamento cirúrgico é recomendado para pacientes cuja citologia for suspeita para malignidade (categoria $\mathrm{V}$ de Bethesda) ou maligna (categoria VI). Frente a uma citologia sugestiva de neoplasia folicular (categoria VI de Bethesda), a cintilografia com radioido é útil para definir a conduta, uma vez que diante de nódulos hipercaptantes, a conduta é mais conservadora e sua retirada não é necessária; já quando o nódulo é hipocaptante está indicada a cirurgia. Se a citologia sugere lesão folicular ou atipia de significado indeterminado (categoria III de Bethesda), nova PAAF pode ser realizada com intervalo de 3-6 meses. Se esse resultado persiste, a cirurgia está indicada em pacientes com alta suspeita clínica ou ultrassonográfica de malignidade ou nódulo $>2 \mathrm{~cm}$. Os demais devem ser acompanhados (ROSÁRIO PW, et al., 2013).

Se a amostra for classificada como categoria I de Bethesda, está recomendada nova punção após 3-6 meses, guiada por US. Se o resultado persiste, indica-se a cirurgia para pacientes com alta suspeita clínica ou ultrassonográfica de malignidade ou nódulo $>2,0 \mathrm{~cm}$. Os demais são acompanhados. E por fim, se a amostra é classificada como categoria II de Bethesda (benigna) mas o nódulo marca uma combinação de achados ultrassonográficos suspeitos para malignidade, está aconselhável a repetição da PAAF, e revela uma taxa de malignidade muito superior a 1-3\% (ROSÁRIO PW, et al., 2013).

As categorias III e IV de Bethesda, que denotam citologia indeterminada, podem ter sua natureza elucidada através dos marcadores moleculares. Pode-se assim, alterar o curso em relação a cirurgia quando marcadores moleculares forem usados (MAIA AL, et al., 2007; ROSÁRIO PW, et al., 2013).

Ainda tendo em vista a abordagem cirúrgica, a extensão da cirurgia é guiada por alguns fatores e características dos nódulos. Indica-se tireoidectomia total para nódulos bilaterais; doença nodular associada a radiação; citologia suspeita para malignidade; nódulo $>4,0 \mathrm{com}$ citologia indeterminada e nódulo $\leq 4,0 \mathrm{~cm}$ com alta suspeita clínica ou ultrassonográfica para neoplasia. Já a lobectomia é indicada na doença nodular unilateral e esporádica quando o nódulo é $\leq 4,0 \mathrm{~cm}$, tem citologia indeterminada e baixa suspeita clínica e ultrassonográfica para câncer; ou ainda, em nódulos com citologia insatisfatória (MAIA AL, et al., 2007; ROSÁRIO PW, et al., 2013).

A abordagem para crianças, adolescentes e gestantes são as mesmas, porém na gestação a cintilografia com radioisótomos está contraindicada. Ainda, se houver necessidade cirúrgica, o segundo trimestre é o melhor período para se realiza-la (ROSÁRIO PW, et al., 2013). Ou seja, em uma investigação cujo diagnóstico seja de neoplasia folicular a opção terapêutica envolve ressecção cirúrgica no segundo trimestre ou realização da investigação com radioisótopo e definição da conduta no período pós-parto. Ainda, deve-se considerar individualmente cada caso, estabelecendo-se acordo entre paciente, endocrinologista e cirurgião (MACIEL e MAGALHÃES, 2008).

$\mathrm{Na}$ avaliação dos nódulos no bócio multinodular leva-se em consideração as características ultrassonográficas sugestivas de malignidade identificadas nos diversos nódulos a fim de definir qual(is) será (ão) puncionado(s). Se nenhum nódulo apresenta aspecto suspeito na US, o risco de malignidade é baixo e apenas o nódulo dominante deve ser aspirado (MAIA AL, et al., 2007).

\section{CONSIDERAÇÕES FINAIS}

Em conclusão, nota-se que é de grande importância o estudo dos nódulos tireoidianos, visto que consiste numa patologia comum na prática clínica, e o conhecimento sobre o assunto leva ao diagnóstico precoce dos nódulos com características sugestivas de malignidade, e assim melhora o prognóstico do paciente. Além disso, o conhecimento permite mais segurança ao lidar com os casos na atuação médica, contribuindo ainda para a melhor relação médico-paciente. 


\section{REFERÊNCIAS}

1. COOPER DS, CHAIR MD, DOHERTY GM, et al. Revised American Thyroid Association Management Guidelines for Patients with Thyroid Nodules and Differentiated Thyroid Cancer. Thyroid 2009; 19(11): 1167-1214.

2. CRISTO AP, GOLDSTEIN HF, FACCIN CS, et al. Increasing diagnostic effectiveness of thyroid nodule evaluation by implementation of cell block preparation in routine US-FNA analysis. Arch Endocrinol Metab. 2016; 60(4): 367-73.

3. KIMURA ET, TINCANI AJ, WARD LS, et al. Doença Nodular da Tireoide: Diagnóstico. Diretrizes Clínicas na Saúde Complementar. Associação Médica Brasileira. 2011; 1-14.

4. MACIEL LMZ, MAGALHÃES PKR. Tireoide e Gravidez. Arq Bras Endocrinol Metab. 2008; 52(7): 1084-1095.

5. MAIA A L, Ward LS, CARVALHO GA, GRAF H, et al. Nódulos de Tireóide e Câncer Diferenciado de Tireóide: Consenso Brasileiro. Arq Bras Endocrinol Metab 2007; 51(5): 867-893.

6. OLIVA EG. PAAF de Tireóide: Correlação Cito-Histológica de 159 casos operados em um hospital terciário. Monografia (Residência Médica em Cirurgia Geral) - Comissão de Residência Médica do Hospital do Servidor Público Municipal, São Paulo, 2012; 20 p.

7. PAZ-FILHO G, GRAF H, WARD LS. Análise comparativa das novas diretrizes e consensos para o manejo do hipotireoidismo, nódulos tireoidianos e câncer diferenciado de tireoide. Arq Bras Endocrinol Metab. 2013; 57(4): 233239.

8. PECCIN S, FURLANETTO TW, CASTRO JAS, et al. Nódulos de Tireóide: Valor da Ultra-Sonografia e da Biópsia por Punção Aspirativa no diagnóstico de câncer. Rev Assoc Med Bras 2003; 49(2): 145-9.

9. ROSÁRIO PW, WARD LS, CARVALHO GA, et al. Nódulo Tireoidiano e Câncer Diferenciado de Tireoide: atualização do Consenso Brasileiro. Arq Bras Endocrinol Metab. 2013; 57(4): 240-64.

10. WARD LS, KLOOS RT. Molecular markers in the diagnosis of thyroid nodules. Arq Bras Endocrinol Metab. 2013; 57(2): 89-97. 